\title{
PD-L1 positivity predicts response
}

Immune-checkpoint inhibitors have substantially improved the outcomes of a subset of patients with non-small-cell lung cancer (NSCLC). However, the lack of a response in many patients, added to the high costs and risks of adverse events, indicates a need for better biomarkers to guide patient selection. Now, the outcomes of an open-label phase II trial, in which patients were stratified by programmed cell death 1 ligand 1 (PD-L1) expression, demonstrate the validity of this biomarker.

A total of 288 patients with recurrent stage IIIB or stage IV NSCLC without detectable EGFR or $A L K$ alterations received 2-weekly nivolumab $(3 \mathrm{mg} / \mathrm{kg})$ plus 6-weekly ipilimumab $(1 \mathrm{mg} / \mathrm{kg})$ for $\geq 2$ years or until disease progression or unacceptable toxicities. The overall response rate (ORR) was $30 \%$ among all patients including complete responses in $2.4 \%$ of patients, with a median progression-free survival (PFS) of 4.2 months. The ORR among patients with PD-L1 expression on $\geq 1 \%$ of tumour cells was $41 \%$ versus $15 \%$ in those with tumour PD-L1 <1\%. Similarly, among the 98 patients with sufficient tumour material available to evaluate tumour mutational burden (TMB), those with a TMB $\geq 10$ mutations per megabase (mut/Mb) had an ORR of $44 \%$ versus $12 \%$ in those with a TMB $<10 \mathrm{mut} / \mathrm{Mb}$. PD-L1 expression did not affect the response rate of patients with a TMB $\geq 10$ mut $/ \mathrm{Mb}$ (ORR $48 \%$ in patients with PD-L1 $\geq 1 \%$ versus $47 \%$ in those with PD-L1 $<1 \%$ ). Grade $\geq 3$ treatment-related adverse effects (TRAEs) were observed in $29 \%$ of patients; $15.6 \%$ of patients discontinued both agents owing to such events.

These findings demonstrate the efficacy of combining immune-checkpoint inhibition in the first-line setting for patients with metastatic NSCLC. The substantially improved response rates of patients with $\geq 1 \%$ PD-L1 expression provide a potential biomarker to guide the selection of patients who are most likely to benefit from this combination.

Peter Sidaway

ORIGINAL ARTICLE Ready, N. et al. First-line nivolumab plus ipilimumab in advanced non-small-cell lung cancer (CheckMate 568): outcomes by programmed death ligand 1 and tumor mutational burden as biomarkers. J. Clin. Oncol. https://doi.org/10.1200/JCO.18.01042 (2019)

\section{OVARIAN CANCER}

\section{Lymphadenectomy is not always required}

Many women undergoing surgery for ovarian cancer also undergo systematic pelvic and para-aortic lymphadenectomy, often despite a macroscopically complete resection with no evidence of lymph node enlargement. Now, data from a randomized controlled trial demonstrate that lymphadenectomy does not improve the outcomes of this patient population.

A total of 1,895 women were assessed for trial eligibility, 1,245 of whom were excluded predominantly owing to incomplete resection, enlarged lymph nodes or other histological findings that met the exclusion criteria. A total of 650 eligible patients were randomly assigned (1:1) to undergo surgery with or without lymphadenectomy. The primary end point was overall survival (OS); secondary end points included progression-free survival (PFS) and quality of life.

No significant differences in OS (HR for death in the lymphadenectomy group 1.06, $95 \%$ $\mathrm{Cl} 0.83-1.34 ; P=0.65$ ) or PFS (HR $1.11,95 \% \mathrm{Cl}$ $0.92-1.34 ; P=0.29$ ) were observed between the groups. However, patients in the no lymphadenectomy group had a decreased risk of almost all post-operative complications, including the need for repeat laparotomy (12.4\% versus $6.5 \%$ of patients; $P=0.01$ ) and risk of death within 60 days of surgery $(3.1 \%$ versus $0.9 \% ; P=0.049$ ). The finding of no significant difference in OS or PFS is of particular interest given that analysis of lymph node specimens from patients in the lymphadenectomy group revealed the presence of microscopic metastases in $55.7 \%$ of patients.

These results contradict the findings of a previous trial, and this difference is largely attributed to the prior prospective evaluation of surgical performance of participating centres and the exclusion of all patients with bulky lymph nodes, for whom lymphadenectomy is clearly necessary. In conclusion, this trial provides level 1 evidence that patients with no obvious signs of lymph node metastases during surgery can safely forego lymphadenectomy.

Peter Sidaway

ORIGINAL ARTICLE Harter, P. et al. A randomized trial of lymphadenectomy in patients with advanced ovarian neoplasms. N. Engl.J. Med. 380, 822-832 (2019)
BREAST CANCER

\section{Fulvestrant enables better outcomes}

\section{Maintenance therapy with aromatase} inhibitors, such as anastrozole, delays or prevents disease progression in a subset of women with metastatic, hormone-receptor (HR)-positive breast cancer; however, the majority of women do not have long-term responses to these agents. Now, new data from a phase III trial (S0226) confirm the superior efficacy of the addition of the selective oestrogen receptor degrader fulvestrant to anastrozole versus anastrozole alone.

A total of 707 postmenopausal women who had not previously received chemotherapy, hormone therapy or immunotherapy for metastatic disease were randomized (1:1) to receive either anastrozole plus fulvestrant or anastrozole alone, with a primary end point of progression-free survival (PFS). After a median follow-up duration of 13.5 months (7 years in those without disease progression), median PFS was 13.5 months among women receiving anastrozole versus 15.0 months in those receiving anastrozole plus fulvestrant (HR 0.81, 95\% Cl 0.69-0.94; $P=0.007$ ). Median overall survival (OS) was 49.8 months in patients receiving anastrozole plus fulvestrant versus 42.0 months among those in the anastrozole group (HR 0.82, 95\% $\mathrm{Cl} 0.69-0.98 ; P=0.03$ ). Data from subgroup analyses revealed no significant differences in OS among any of the subgroups, including those stratified by endocrine sensitivity, previous endocrine therapy, measurable disease or disease location.

Few new adverse events emerged in the extended follow-up period: patients in the anastrazole plus fulvestrant group had a slightly increased risk of grade $\geq 3$ adverse events ( $15 \%$ versus $13 \% ; P=0.47$ ), including musculoskeletal pain, fatigue and hot flushes. No new grade 4 or 5 adverse events were reported.

These data support the routine clinical use of anastrozole plus fulvestrant in women with HR-positive metastatic breast cancer. Notably, this trial provided a robust efficacy signal despite a substantial number of women (45\%) crossing over to the anastrozole plus fulvestrant group, as permitted by the trial design.

Peter Sidaway

ORIGINAL ARTICLE Mehta, R. S. et al. Overall survival with fulvestrant plus anastrozole in metastatic breast cancer. N. Engl.J. Med. 380, 1226-1234 (2019) 